\title{
Establishment of a transient transfection system for Babesia sp. Xinjiang with the use of homologous promoters
}

\section{Xiaoxing Wang}

Lanzhou Veterinary Research Institute Jinming Wang

Lanzhou Veterinary Research Institute Junlong Liu

Lanzhou Veterinary Research Institute

Jifei Yang

Lanzhou Veterinary Research Institute

\section{Zhaoyong}

Lanzhou Veterinary Research Institute

\section{Aihong Liu}

Lanzhou Veterinary Research Institute

\section{Youquan Li}

Lanzhou Veterinary Research Institute

\section{Yubin Li}

Lanzhou Veterinary Research Institute

\section{Haiou Lan}

Lanzhou Veterinary Research Institute

\section{Guangyuan Liu}

Lanzhou Veterinary Research Institute Jianxun Luo

Lanzhou Veterinary Research Institute

Guiquan Guan ( $\square$ guanguiquan@caas.cn )

Lanzhou Veterinary Research Institute

Hong Yin

Lanzhou Veterinary Research Institute

\section{Short Report}

Keywords: Babesia sp. Xinjiang, transient transfection, promoter activity, genetic manipulation 
Posted Date: October 15th, 2020

DOl: https://doi.org/10.21203/rs.3.rs-90736/v1

License: (c) (i) This work is licensed under a Creative Commons Attribution 4.0 International License. Read Full License 


\section{Abstract}

\section{Background}

Babesia species, the agentic pathogens of human and animal babesiosis, are spread worldwide. Over the last decade, genetic manipulation approaches have been applied with many protozoan parasites, including Plasmodium falciparum, Trypanosoma cruzi, Cryptosporidium parvum, Theileria annulata, Theileria parva, Babesia bovis, Babesia bigemina, Babesia ovata, Babesia gibsoni, and Babesia ovis. For Babesia sp. Xinjiang $(B x j)$, which is the causative pathogen of ovine babesiosis mainly in China, the efficiency of these techniques remain unclear.

Methods

First, a plasmid bearing the elongation factor- 1 alpha promoter, as well as the firefly luciferase reporter gene and rap stop region were transfected into $B x j$ by electroporation and nucleoporation to determine the most suitable transfection solution. Then, six program setting were evaluated to confirm the best for $B x j$ transient transfection and a series of different amounts of plasmid DNA were applied to generate relatively high luminescence values. Finally, the activities of four promoters derived from $B x j$ were evaluated using the developed transient transfection system.

\section{Conclusions}

In this study, a transient transfection system for $B x j$ was optimized. These findings provided critical information for $B x j$ genetic manipulation, as an essential tool to identify virulence factors and to further elucidate the basic biology of pathogens.

\section{Background}

Babesiosis, caused by apicomplexan intraerythrocytic protozoan pathogens of the genus Babesia infective to humans, as well as domestic and wild animals, is a re-emerging tick-borne disease in tropical and subtropical regions worldwide [1]. Since the first documented Babesia species was reported in 1888, more than 100 others have been identified globally, although few (Babesia ovis, Babesia motasi, and Babesia crassa) have been confirmed as causative agents of ovine babesiosis. This group of Babesia spp. has received much attention since $B$. ovis is the main etiological agent of ovine babesiosis, while $B$. motasi and $B$. crassa have been implicated in human cases of babesiosis throughout Asia [2-6]. Additionally, a novel Babesia species, named Babesia sp. Xinjiang (Bxj), experimentally transmitted by Hyalomma anatolicum anatolicum (Koch, 1844), was first isolated from a sheep bitten by Rhipicephalus sanguineus and $H$. anatolicum anatolicum [7]. Epidemiological investigations revealed that the seropositivity rate of $B x j$ ranged from 0 to $68.13 \%$ in 22 provinces across China [8]. Ovine babesiosis is an important socioeconomic factor, particularly in animal mortality, costs of livestock treatment, and productivity loss [9]. 
The difficulty in controlling ovine babesiosis is due to the lack of effective vaccines, limited choices of therapeutic drugs, and the emergence of several drug-resistant Babesia strains. Understanding the basic biology of Babesia species is pivotal to the development of effective therapeutic drugs and preventive vaccines to control this disease [10]. Genetic manipulation tools are considered as effective measures to identify virulence factors of particular agents, understand host-parasite interactions, and elucidate the mechanisms underlying drug resistance [11]. Such techniques have been described in several protozoan parasites, including Plasmodium falciparum, Toxoplasma gondii, Cryptosporidium parvum, Theileria annulata, Babesia bovis, Babeisa bigemina, Babeisa ovata, Babesia gibsoni, and Babesia ovis [12-21]. Mapping and sequencing of the $B x j$ genome were completed by our laboratory (Vector and Vector-Borne Diseases Laboratory, Lanzhou Veterinary Research Institute) in 2006. In this study, a suitable transfection system for Bxj merozoites was developed using electroporation and nucleofactor technologies.

\section{Methods}

\section{In vitro culture of Babesia sp. Xinjiang}

A monoclonal line (G5) of $B x j$ was cultured in vitro as reported previously [22]. Briefly, when parasitemia reached $20 \%-30 \%, 4 \mathrm{~mL}$ of a suspension of infected red blood cells (RBCs) were cultured in a T75 flask supplemented with $5 \%$ fresh sheep erythrocytes and $20 \%$ fetal bovine serum (Gibco, Carlsbad, CA, USA) in Roswell Park Memorial Institute 1640 medium (Lonza Biologics, Portsmouth, NH, USA) at $37^{\circ} \mathrm{C}$ under an atmosphere of $5 \% \mathrm{CO}_{2} / 95 \%$ air.

\section{Plasmid constructs}

Schematic diagrams of the transient transfection plasmids used in this study are presented in Fig. 1a and 1b. Five distinct plasmids were generated, named as phosphate-buffered saline (PBS)-LuRaA, PBSLuRaB, PBS-LuRaAc, and PBS-LuRaRa. Initially, the plasmid PBS-LuRa was constructed by amplifying the firefly luciferase gene and $B x$ j rhoptry-associated protein-1 3'-flasking region (rap3') by PCR from the plasmid pENT12luc and genomic DNA of Bxj, respectively, using specific primer pairs (Table 1). The PCR products were inserted into the Xho I restriction site of the plasmid pBluescript SK(+) (provided by Carlos E. Suarez) using the MultiS One Step Cloning Kit (Vazyme Biotech Co., Ltd., Nanjing, China). Then, the promotors of $B x j$ ef1 a IG-A, ef-1 a IG-B, actin 5'-untranslated region (UTR), and rap 5'-UTR were amplified by PCR and cloned into the Sall restriction site of PBS-LuRa, respectively. A free-promoter plasmid (PBSLuRa) was used as a negative control. The constructed plasmids were confirmed by sequencing and purified using the QIAGEN Plasmid Maxi Kit (Qiagen, Hilden, Germany) in accordance with the manufacturer's instructions.

\section{Optimization of parasite transfection}

When parasitemia of $B \times j$-infected RBCs reached $25 \%-30 \%$, the cultures were centrifuged at $800 \times g$ for 10 min. Then, the supernatant was discarded and pellets of $B x$ jinfected RBCs were washed twice in PBS and once in cytomix buffer $\left(120 \mathrm{mM} \mathrm{KCl}, 0.15 \mathrm{mM} \mathrm{CaCl}_{2}, 10 \mathrm{mM} \mathrm{K} \mathrm{HPO}_{4} / \mathrm{KH}_{2} \mathrm{PO}_{4}, \mathrm{pH} 7.6,25 \mathrm{mM}\right.$ 
HEPES. pH7.6, 2 mM EGTA, $5 \mathrm{mM} \mathrm{MgCl}_{2}$, final pH 7.6) [14]. Parasite transfections were performed with the use of a Gene Pulser Xcell ${ }^{\mathrm{TM}}$ Electroporation System (Bio-Rad Laboratories, Hercules, CA, USA) and 0.2-cm cuvettes containing $50 \mu \mathrm{L}$ of sterilized cytomix buffer or $20 \mu \mathrm{g}$ of plasmids and $1 \times 10^{8}$ infected RBCs to a final volume of $100 \mu \mathrm{L}$. Initially, two types of nucleofection buffer (human T cell Nucleofector solution and Basic Parasite Nucleofector ${ }^{\circledR}$ Solution) were used to screen the optimization buffer with the program settings U-033, V-024, X-001, D-023, T-023, and T-020. Cytomix buffer was used for electrotransfection at $1200 \mathrm{~V}$ and $25 \mu \mathrm{F}$. Subsequently, optimized parameters for $B \times j$ transfection were employed to determine the amounts of plasmid, which varied from 5 to $150 \mu \mathrm{g}$. After transfection, the mixtures were transferred into the wells of a 24-well culture plate containing 7.5\% fresh sheep RBCs and $10 \%$ fetal bovine serum. The luciferase activities and percentage of parasitized erythrocytes (PPEs) were determined at 24,48 , and $72 \mathrm{~h}$ post-transfection. Then, $20 \mu \mathrm{g}$ of each constructed plasmid (PBS-LuRaA, PBS-LuRaB, PBS-LuRaAc, and PBS-LuRaRa) were transfected into infected RBCs using the optimized $B x j$ transfection conditions. Promoter activity was evaluated with the use of a ONE-GloTM Luciferase Assay System (Promega Corporation, Madison, WI, USA) at 24, 48, and $72 \mathrm{~h}$ post-transfection. Briefly, $100 \mu \mathrm{L}$ of ONE-Glo ${ }^{\mathrm{TM}}$ Luciferase Assay Reagent was mixed with $100 \mu \mathrm{L}$ of transfected $B x j$ culture in the wells of white 96-well plates and then incubated for $5 \mathrm{~min}$ at room temperature. Afterward, the chemiluminescence values were determined at 10-s integration intervals using a GloMax®-Multi Detection System (Promega Corporation).

\section{Results And Discussion}

Optimization of $B x j$ merozoite transfection parameters

Experimental conditions for transit transfection of $B x j$ merozoites were determined using a Gene Pulser Xcel $^{\mathrm{TM}}$ Electroporation System and a Nucleofector ${ }^{\mathrm{TM}} \mathrm{II} / 2 \mathrm{~b}$ Transfection Device (Lonza). To confirm the suitability of the transient transfection solution, luciferase activity and PPEs were determined at 24,48 , and $72 \mathrm{~h}$, when $20 \mu \mathrm{g}$ of plasmid DNA with each buffer (human T cell nucleofector solution and Basic parasite nucleofector solution) were transfected with the V-024 program setting, and while the cytomix buffer was electrotransfected with $20 \mu \mathrm{g}$ of PBS-LuRaB at $1200 \mathrm{~V}$ and $25 \mu \mathrm{F}$ (Fig. 1c). As compared to the other two solutions, the luciferase activity of the transfection system with human $\mathrm{T}$ cell nucleofector solution was relatively high $\left({ }^{\star \star} p<0.001\right)$, indicating that this solution was suitable for $B x j$ transient transfection. With the exception of the cytomix buffer, there was no decrease in relative luciferase activity in the human T cell nucleofector solution or basic parasite nucleofector solution over a period of $72 \mathrm{~h}$. In previous reports, there was a downward trend in relative luciferase activity during transfection of $B$. bovis, $B$. ovis, and Babesia microti. One possible explanation for this exceptional situation was that human $T$ cell nucleofector solution and basic parasite nucleofector solution were more capable of conveying protective effects to the ovine erythrocyte membrane.

In terms of the impact of each transfection buffer to PPEs, a significant high figures using basic parasite nucleofector solution can be obtained at $72 \mathrm{~h}$ post-transfection compared to other transfection solutions (Fig. 1d), while the highest level of relative luminescence units observed in human T cell nucleofector 
solution group instead of cytomix and basic parasite nucleofector solution groups (Fig. 1c). These data provide additional evidence that human $T$ cell nucleofector solution was more effective and suitable for transient transfection of $B x j$.

Furthermore, as compared to the other five program settings (U-033, X-001, D-023, T-023, and T-020), luciferase activity was significantly greater with V-024 $\left.{ }^{\star \star \star *} p<0.0001\right)$. Relative luciferase activities at 24 , 48, and $72 \mathrm{~h}$ post-transfection are presented in Fig. 2e. Subsequently, human T cell Nucleofector solution and V-024 program were used as transient transfection parameters to determine the amount of plasmid DNA. With an increase in the amount of plasmid DNA, relative luminescence units tended to increase, while almost similar values were observed with 20,50 , and $100 \mu \mathrm{g}(p>0.05)$ (Fig. 1f). As compared to the use of $10 \mu \mathrm{g}, 20 \mu \mathrm{g}$ generated a relatively high relative light unit value $\left({ }^{*} p<0.05\right)$. Taken together, these results, including relative light units and PPEs, showed that human T-cell solution, the program V024 , and $20 \mu \mathrm{g}$ of plasmid DNA were the most favorable conditions for transient transfection in subsequent studies.

Evaluation of promoter activity

To determine promoter activity, the optimal transfection conditions mentioned above, including the transfection program, buffer, and amount of plasmid DNA, were employed to transfect $B x j$ merozoites with five types of plasmids (PBS-LuRaA, PBS-LuRaB, PBS-LuRaAc, PBS-LuRaRa and PBS-LuRa). The time courses of luciferase activities in the transfected Bxj culture are presented in Fig. 2a. Although four of the promoters tested in this study were able to promote luciferase expression, a significantly higher level of luciferase activity was observed with transfection of the $B \times j$ merozoites with either plasmid PBS-LuRaAc or PBS-LuRaB after electroporation. According to values of luciferase activity post-transfection, the extent of promoter activity can be classified as strong (actin $5^{\prime}$ UTR), medium (ef1 a IG-B), or low (ef1a IG-A and rap 5'-UTR). The PPEs of each group at 24,48 , and $72 \mathrm{~h}$ post-transfection were determined by calculating the proportion of infected RBCs out of 2000 cells under a light microscope. There were no obvious difference in PPE values among the four groups at 24,48 , and $72 \mathrm{~h}$ post-transfection $(p>0.05)$ (Fig. $2 \mathrm{~b})$.

\section{Conclusions}

In conclusion, the conditions for transient transfection of $B x j$, including the transfection solution, program settings, and quality of plasmid DNA, were optimized. In addition, promoter activity was systematically evaluated. This study is the first effort to create a platform for transient transfection of $B x j$ and provides basic information for the development of a stable transfection method for $B x j$ and to further elucidate the biology of this parasite, as well as the parasite-vector and parasite-host interactions.

\section{Abbreviations}

Bxj: Babesia sp. Xinjiang

RBCs: Red blood cells 
PCR: Polymerase chain reaction

\section{Declarations}

\section{Ethical Approval and Consent to participate}

The study was approved by the Animal Ethics Committee of the Lanzhou Veterinary Research Institute, CAAS (Permit No. LVRIAEC-2018-001). All the procedures were conducted according to the Animal Ethics Procedures and Guidelines of the People's Republic of China.

\section{Consent for publication}

All authors consent to be published.

\section{Availability of supporting data}

All data are available upon request.

\section{Competing interests}

The authors declare no conflict of interest.

\section{Funding}

This study was financially supported by the National Science Foundation of China (grant nos. 31972701 and 31972706), National Key Research and Development Program of China (grant no.

2017YFD0501200), the 973 Program (grant no. 2015CB150300), ASTIP (grant no. CAAS-ASTIP-2016-

LVRI), NBCIS (grant no. CARS-37), and the Jiangsu Co-innovation Center Program for the Prevention and Control of Important Animal Infectious Disease and Zoonoses.

\section{Authors' contributions}

$X W$ and JW carried out the experiments. JW wrote the draft of the manuscript. JL, JY, ZL, AL, YL, YuL and HL participate plasmid construction. GG corrected the manuscript. GL, JLuo and HY supervised all parts of the study. All authors have read and approved the final version of this manuscript.

\section{Acknowledgements}

We acknowledge Carlos E. Suarez for technical support and providing pBluescript SK(+) plasmid.

\section{Authors' information}

Xiaoxing Wang, 1317212955@qq.com

Jinming Wang,wjm0403@caas.cn; 
Junlong Liu, liujunlong@caas.cn;

Jifei Yang, yangjifei@caas.cn;

Zhaoyong Lv,807960565@qq.com

Aihong Liu, liuaihong@caas.cn

You-quan Li, liyouquan@caas.cn

Yubin Li, 3213447783@qq.com

Haiou Lan,1327633849@qq.com

Guangyuan Liu, liuguangyuan@caas.cn

Jianxun Luo, luojianxun@caas.cn

Gui-quan Guan, guanguiquan@caas.cn;

Hong Yin, yinhong@caas.cn

\section{References}

1 Uilenberg G. International collaborative research: Significance of tick-borne hemoparasitic diseases to world animal health. Vet Parasitol 1995;57(1-3):19-41.

2 Kim JY, Cho SH, Joo HN, Tsuji M, Cho SR, Park IJ et al. First case of human babesiosis in korea: Detection and characterization of a novel type of babesia sp. (ko1) similar to ovine babesia. J Clin Microbiol 2007;45(6):2084-7.

3 Hong SH, Kim SY, Song BG, Rho JR, Cho CR, Kim CN et al. Detection and characterization of an emerging type of babesia sp. Similar to babesia motasi for the first case of human babesiosis and ticks in korea. Emerg Microbes Infect 2019;8(1):869-78.

4 Man SQ, Qiao K, Cui J, Feng M, Fu YF and Cheng XJ. A case of human infection with a novel babesia species in china. Infect Dis Poverty 2016;5(28.

5 Jiang JF, Zheng YC, Jiang RR, Li H, Huo QB, Jiang BG et al. Epidemiological, clinical, and laboratory characteristics of 48 cases of "babesia venatorum" infection in china: A descriptive study. Lancet Infect Dis 2015;15(2):196-203.

6 Wang J, Zhang S, Yang J, Liu J, Zhang D, Li Y et al. Babesia divergens in human in gansu province, china. Emerg Microbes Infect 2019;8(1):959-61. 
7 Guan G, Ma M, Moreau E, Liu J, Lu B, Bai Q et al. A new ovine babesia species transmitted by hyalomma anatolicum anatolicum. Exp Parasitol 2009;122(4):261-7.

8 Guan G, Ma M, Liu A, Ren Q, Wang J, Yang J et al. A recently identified ovine babesia in china: Serology and sero-epidemiology. Parasitol Int 2012;61(4):532-7.

9 Aouadi A, Leulmi H, Boucheikhchoukh M, Benakhla A, Raoult D and Parola P. Molecular evidence of tickborne hemoprotozoan-parasites (theileria ovis and babesia ovis) and bacteria in ticks and blood from small ruminants in northern algeria. Comp Immunol Microbiol Infect Dis 2017;50(34-39.

10 Antunes S, Rosa C, Couto J, Ferrolho J and Domingos A. Deciphering babesia-vector interactions. Front Cell Infect Microbiol 2017;7(429.

11 Suarez CE, Bishop RP, Alzan HF, Poole WA and Cooke BM. Advances in the application of genetic manipulation methods to apicomplexan parasites. Int J Parasitol 2017;47(12):701-10.

12 Padmanabhan PK, Polidoro RB, Barteneva NS, Gazzinelli RT and Burleigh BA. Transient transfection and expression of foreign and endogenous genes in the intracellular stages of trypanosoma cruzi. Mol Biochem Parasitol 2014;198(2):100-3.

13 Suarez CE and McElwain TF. Stable expression of a gfp-bsd fusion protein in babesia bovis merozoites. Int J Parasitol 2009;39(3):289-97.

14 Asada M, Tanaka M, Goto Y, Yokoyama N, Inoue N and Kawazu S. Stable expression of green fluorescent protein and targeted disruption of thioredoxin peroxidase-1 gene in babesia bovis with the wr99210/dhfr selection system. Mol Biochem Parasitol 2012;181(2):162-70.

15 Vinayak S, Pawlowic MC, Sateriale A, Brooks CF, Studstill CJ, Bar-Peled Y et al. Genetic modification of the diarrhoeal pathogen cryptosporidium parvum. Nature 2015;523(7561):477-80.

16 Adamson R, Lyons K, Sharrard M, Kinnaird J, Swan D, Graham S et al. Transient transfection of theileria annulata. Mol Biochem Parasitol 2001;114(1):53-61.

17 De Goeyse I, Jansen F, Madder M, Hayashida K, Berkvens D, Dobbelaere D et al. Transfection of live, tick derived sporozoites of the protozoan apicomplexan parasite theileria parva. Vet Parasitol 2015;208(34):238-41.

18 Hakimi H, Yamagishi J, Kegawa Y, Kaneko O, Kawazu S and Asada M. Establishment of transient and stable transfection systems for babesia ovata. Parasit Vectors 2016;9(171.

19 Silva MG, Knowles DP and Suarez CE. Identification of interchangeable cross-species function of elongation factor-1 alpha promoters in babesia bigemina and babesia bovis. Parasit Vectors 2016;9(1):576. 
20 Liu M, Asada M, Cao S, Adjou Moumouni PF, Vudriko P, Efstratiou A et al. Transient transfection of intraerythrocytic babesia gibsoni using elongation factor-1 alpha promoter. Mol Biochem Parasitol 2017;216(56-59.

21 Rosa C, Asada M, Hakimi H, Domingos A, Pimentel M and Antunes S. Transient transfection of babesia ovis using heterologous promoters. Ticks Tick Borne Dis 2019;10(6):101279.

22 Guan GQ, Ma ML, Liu AH, Du PF, Ren QY, Li YQ et al. Continuous in vitro cultivation of a recently identified babesia that infects small ruminants in china. Veterinary Parasitology 2012;187(3-4):371-78.

\section{Tables}

Table 1. The information of primers used in this study

\begin{tabular}{|c|c|c|c|}
\hline Element & Primers & Sequence $\left(5^{\prime}-3^{\prime}\right)$ & $\begin{array}{l}\text { Size } \\
\text { (bp) }\end{array}$ \\
\hline \multirow{2}{*}{$\begin{array}{l}\text { firefly luciferase } \\
\text { gene }\end{array}$} & luc- $F$ & GGTACCGGGCCCCCCCTCGAGatggaagatgccaaaaac & \multirow[t]{2}{*}{1653} \\
\hline & luc- $R$ & TCGATACCGTCGACCTCGAGttacacggcgatcttgccg & \\
\hline \multirow[t]{8}{*}{ Promoter } & ef1a IG A-F & CGAATTGGGTACCGGGCCCCGATAATACTAGGGTTATTG & \multirow[t]{2}{*}{897} \\
\hline & ef1a IG $A-R$ & CCATCTCGAGGGGGGGCCCTTTCGTGGAGTTTTAACTAG & \\
\hline & ef1a IG $B-F$ & GCGAATTGGGTACCGGGCCCCGAAAGGAAAGGTAACGGC & \multirow[t]{2}{*}{958} \\
\hline & ef1a IG $B-R$ & CCATCTCGAGGGGGGGCCCTTTCGTGGAGTTTTAACTAG & \\
\hline & $\operatorname{rap}_{F} 5^{\prime} U R T-$ & GGCGAATTGGGTACCGGGCCCGAGGATGAGGTATCTCAG & \multirow[t]{2}{*}{1386} \\
\hline & $\begin{array}{l}\operatorname{rap} 5^{\prime} U R T- \\
\mathrm{R}\end{array}$ & CCATCTCGAGGGGGGGCCCTCTTAACACCAGGCAATGAA & \\
\hline & $\begin{array}{l}\operatorname{actin} 5^{\prime} \\
\text { UTR-F }\end{array}$ & GGCGAATTGGGTACCGGGCCCAAGTGTCCCAGTTGCCTC & \multirow[t]{2}{*}{2489} \\
\hline & $\begin{array}{l}\operatorname{actin} 5^{\prime} \\
\text { UTR-R }\end{array}$ & CCATCTCGAGGGGGGGCCCTTTTCAAAAAATTCTGTG & \\
\hline \multirow[t]{2}{*}{ Terminator } & $\underset{F}{\operatorname{rap}} 3^{\prime}$ UTR- & GCTTATCGATACCGTCGACGCAGCTTCAAAGCGTACAT & \multirow[t]{2}{*}{1362} \\
\hline & ${ }_{R}^{r a p 3^{\prime}}$ UTR- & GGGCCCCCCCTCGAGGTCGACTCTTAACACCAGGCAATGA & \\
\hline
\end{tabular}

Restriction enzyme sites are underlined

\section{Figures}


a

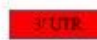

b

PBS-LuRaA ef-1alG-A

PBS-LURaB ef-1aाG-B

PBS-LURaAC actin 5 OTR

PBS-LURaRa $\longrightarrow$ rap

PBS-LuRa

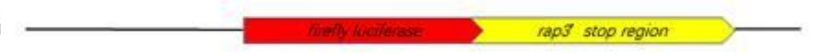

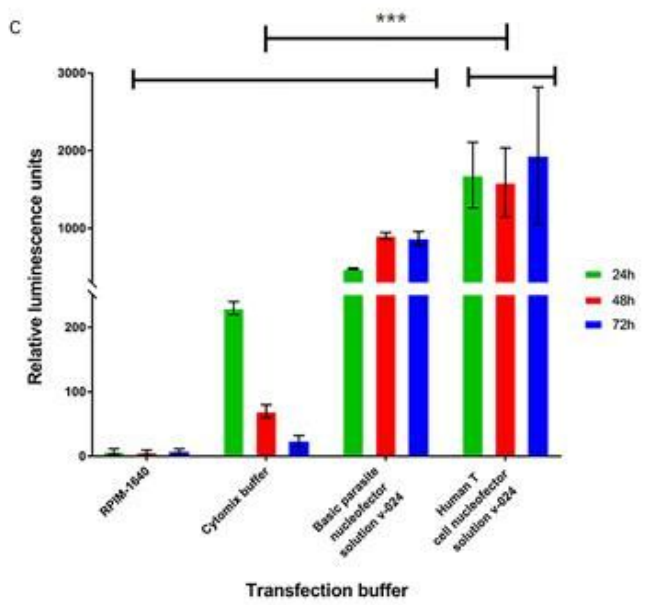
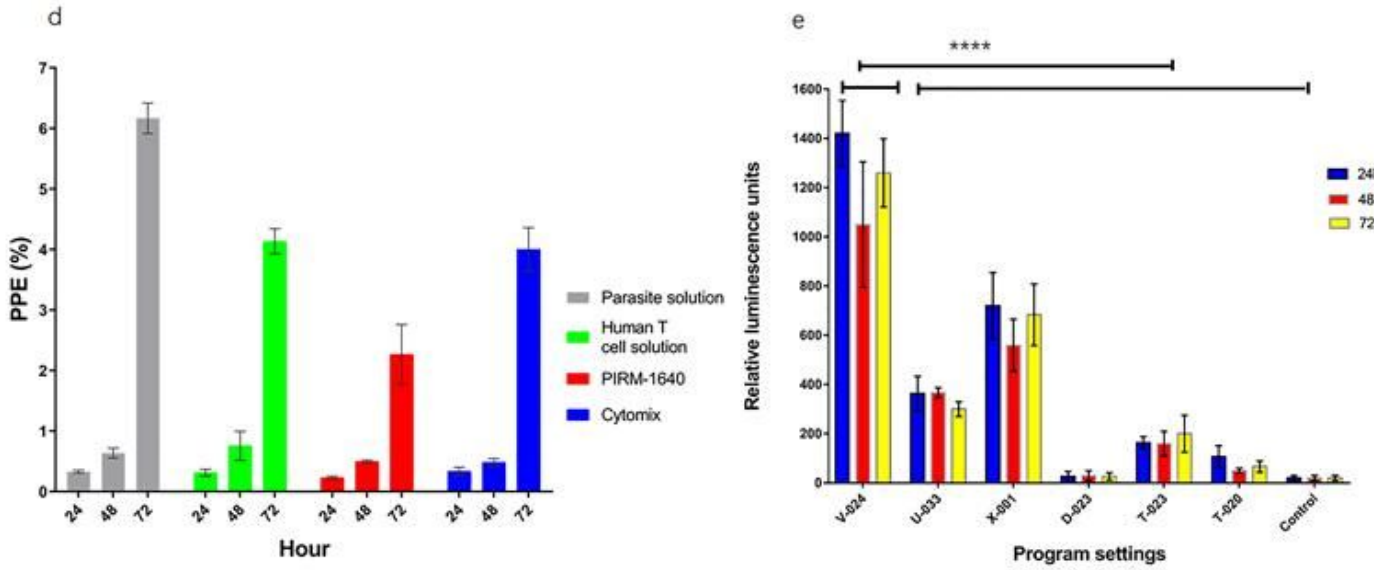

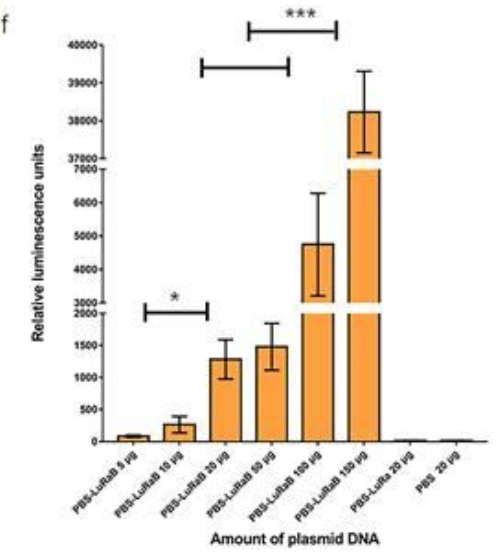

Figure 1

Optimization of the Bxj transient transfection parameters. (a) A diagram of the Bxj ef-1a locus structure. (b) The structures of five types of plasmids used for transient transfection. (c) Transfection buffer optimization by electroporation and nucleofection (V-024 program setting). (d) The time course of PPEs was determined after transfection. (e) The optimization of program settings for Bxj transient transfection. (f) Determination of the optimal amount of plasmid DNA for Bxj transfection. 
a

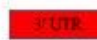

b

PBS-LuRaA ef-1alG-A

PBS-LURaB ef-1aाG-B

PBS-LURaAC actin 5 OTR

PBS-LURaRa $\longrightarrow$ rap

PBS-LuRa

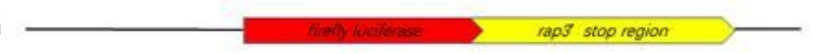

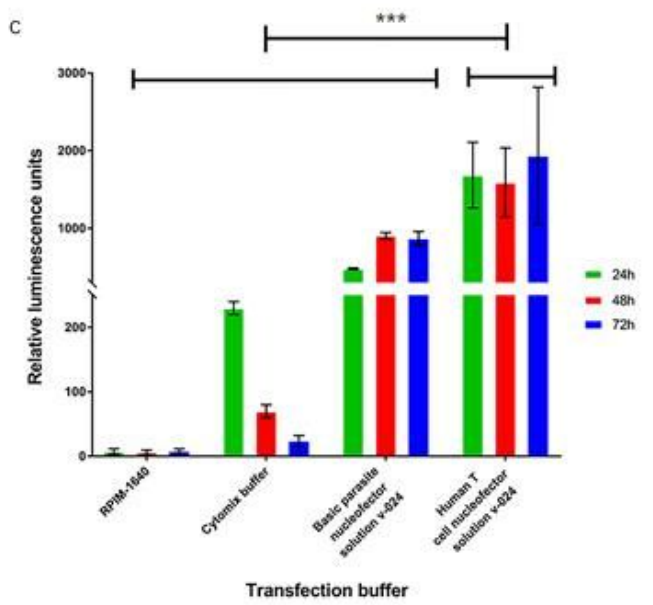
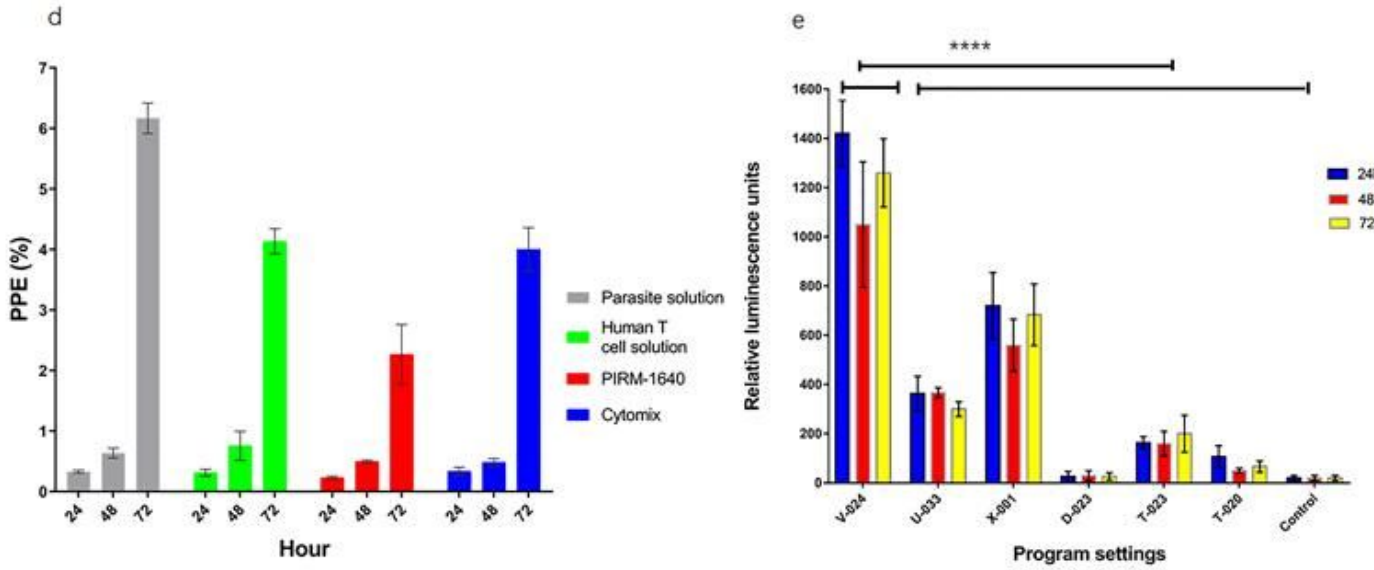

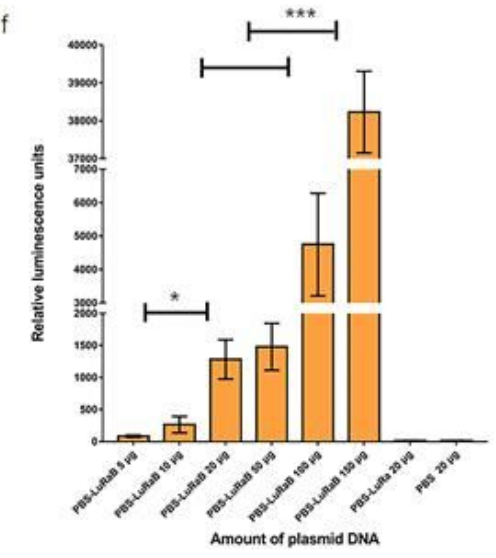

Figure 1

Optimization of the Bxj transient transfection parameters. (a) A diagram of the Bxj ef-1a locus structure. (b) The structures of five types of plasmids used for transient transfection. (c) Transfection buffer optimization by electroporation and nucleofection (V-024 program setting). (d) The time course of PPEs was determined after transfection. (e) The optimization of program settings for Bxj transient transfection. (f) Determination of the optimal amount of plasmid DNA for Bxj transfection. 
a

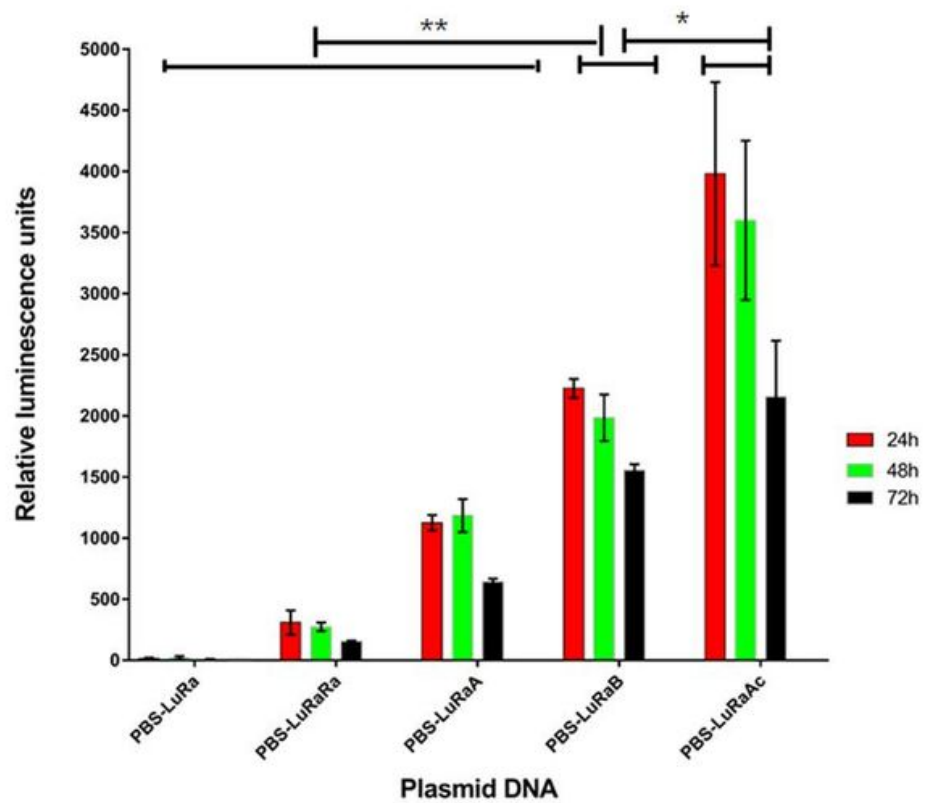

b

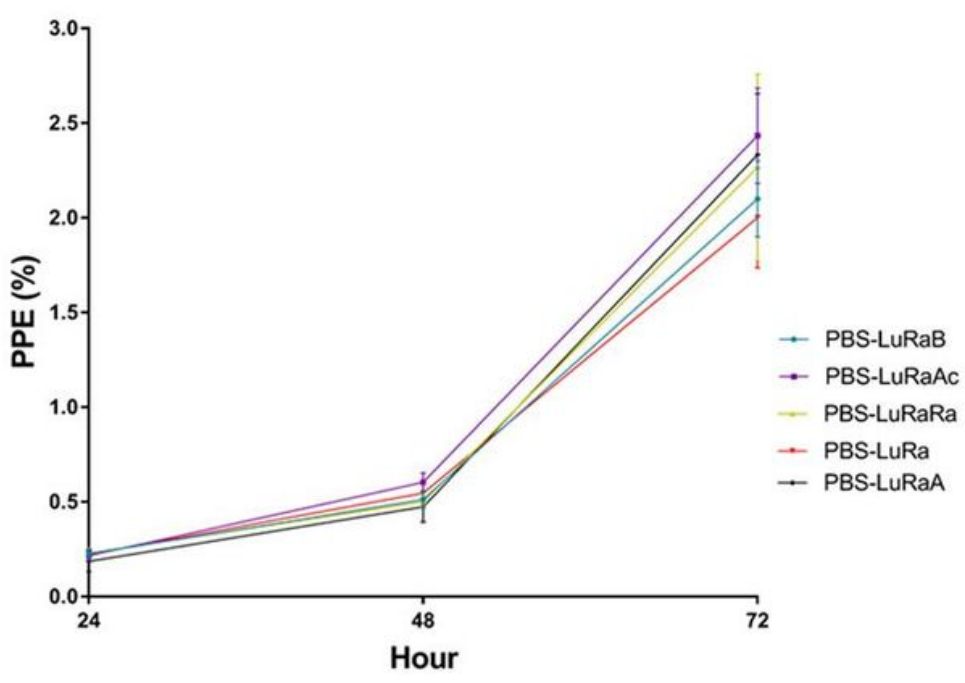

Figure 2

Promoter activities. (a) Evaluation of promoter activities with optimized transfection conditions. (b) PPEs at 24,48 , and $72 \mathrm{~h}$ post-transfection of each type of plasmid DNA bearing different promoters.

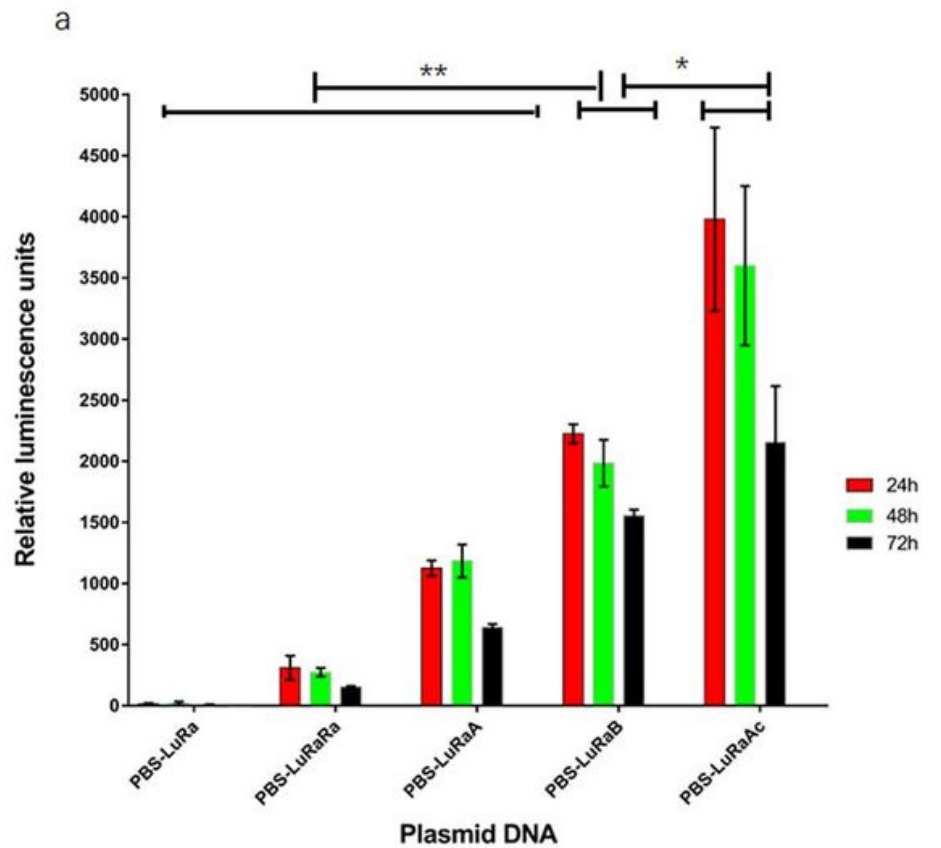

b

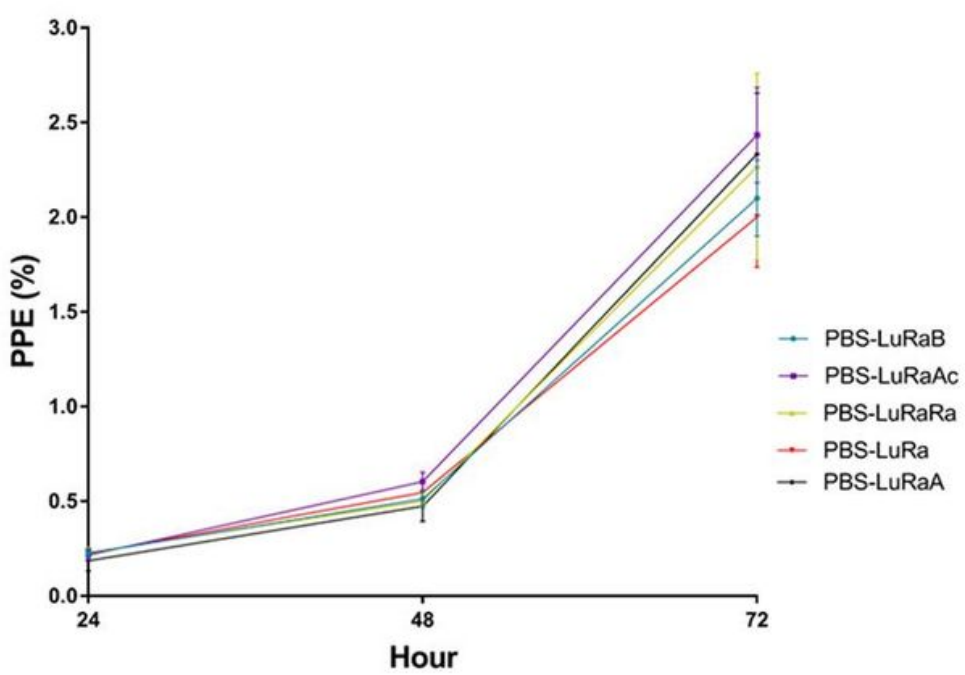

Figure 2

Promoter activities. (a) Evaluation of promoter activities with optimized transfection conditions. (b) PPEs at 24,48 , and $72 \mathrm{~h}$ post-transfection of each type of plasmid DNA bearing different promoters. 